Physics Vol. 2, No. 1, pp. 21-37, 1965. Physics Publishing Co. Printed in Great Britain.

\title{
О РАССЕЯНИИ ЭЛЕКТРОНОВ В МЕТАЛЛЕ НА МАГНИТНЫХ ПРИМЕСНЫХ АТОМАХ И ОСОБЕННОСТЯХ ПОВЕДЕНИЯ СОПРОТИВЛЕНИЯ
}

\author{
A. A. АБРИКОСОВ
}

(Получили: 17 февраля 1965)

\section{А Н Н О Т А ИЯ}

С помощью особой техники квантовой теории поля для спинов произведено вычисление электрического сопротивления металлов с парамагнитными примесями в предположении $J / \epsilon_{F} \ll 1$, но при произвольной величине $\left(J / \epsilon_{\boldsymbol{F}}\right) \ln \left(\epsilon_{\boldsymbol{F}} / T\right)$, где $J$ - амплитуда обменного рассеяния, $\epsilon_{\boldsymbol{F}}-$ энергия Ферми. Первый член этого ряда был ранее найден Кондо [I]. Показано, что обменное и обычное взаимодействия дают независимые слагаемые в сопротивлении. При ферромаГнитном взаимодействии спинов электрона и примеси $(J>0)$ обменная часть сопротивления уменьшается с температурой и исчезает при $T=0$. В обратном случае $(J<0)$ сопротивление при понижении температуры сначала растет. После прохождения максимума (при $T=T_{\max }$ ), где обменное сопротивление, отнесенное к одному атому примеси, сравнивается по порядку величины с обычным, обменное сопротивление и в этом случае уменьшается до нуля при $T=0$. Такое поведение связано резонансным характером амплитуды рассеяния при $J<0$. В расчете предполагается, что спины примесей полностью неупорядочены, т.е. температура выше температуры Кюри примесного ферромагнетизма. Поскольку последняя пропорциональна концентрации, а $T_{\max }$ от нее не зависит, то полученные результаты справедливы при достаточно малых концентрациях.

Появление минимума в электрическом сопротивлении некоторых металлов, как функции температуры уже давно привлекало к себе внимание. Исследование этого явления показало, что по всей вероятности это явление связано с наличием в металле (немагнитном) примесных атомов с незаполненными внутренними оболочками. Однако, до недавнего времени не существовало удовлетворительного объяснения взаимодействия электронов с такими атомами и причин возникновения минимума. Такое объяснение дала работа Кондо [1], в которой была вычислена поправка следующего приближения (по сравнению с борновским) к вероятности рассеяния электронов примесью, связанного с обменным взаимодействем $J$. При этом оказалось, что в вероятности рассеяния появляется

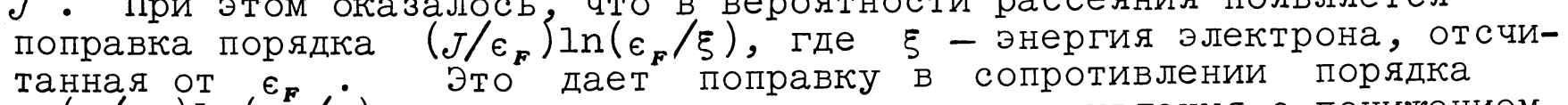
- $\left(J / \epsilon_{F}\right) \ln \left(\epsilon_{\boldsymbol{F}} / \dot{T}\right)$, что и приводит к росту сопротивления с понижением температуры при отрицательном знаке $\mathcal{J}$ (антиферромагнитное взаимодействие электронов с примесью). 
Расчет Кондо справедлив, при двух ограничениях. Во-первых поправка должна быть невелика. Во-вторых спины атомов примеси должнь быть некоррелированы друг с другом. Иначе говоря, температура должна быть выше точки Кюри примесного ферромагнетизма (или антиферромагнетизма). Как известно (см. например [2]), температура Кюри про порциональна концентрации дефектов. В то же время относительная поправка в сопротивлении, пропорциональная $\left(J / \epsilon_{F}\right) \ln \left(\epsilon_{F} / T\right)$ не зависит от концентрации. Следовательно возможна столь малая концентрация дефектов, при которой $\left(J / \epsilon_{F}\right) \ln \left(\epsilon_{F} / T\right)$ становится порядка или больше единицы еще до наступления упорядочения спинов. В этом случае теория возмущений уже неприменима, и необходимо суммировать весь ряд. Это вычисление и проделано в настоящей работе.

\section{1. Спиновая техника}

основная трудность расчета связана с некоммутативностью проекций оператора спина примеси. Отметим, что согласно [1] именно это и приводит к логарифмическим членам. Это означает, что гамильтониан обменного воздействия

$$
H_{\mathrm{int}}=-(J / N) \sum_{n} \psi_{\alpha}{ }^{+}\left(\vec{r}_{n}\right) \vec{\sigma}_{\alpha \alpha^{\prime}} \hat{\vec{S}}_{n} \psi_{\alpha} \cdot\left(\vec{r}_{n}\right)
$$

(взаимодействие считаем 8-функционным) существенно неэквивалентен воздействию спина эпектронов с внешним магнитным полем. С другой стороны операторы $\hat{\vec{S}}_{\mathrm{n}}$ нельзя рассматривать и как обычные операторы поля [3] • Дело в том, что каждое среднее типа

$$
<T \cdot\left(S^{i}\left(t_{1}\right) S^{k}\left(t_{2}\right) \ldots S^{l}\left(t_{n}\right)\right)>
$$

где все $\hat{S}^{1}$ относятся к одному атому, ( $i$ - индекс проекции), $\langle\ldots\rangle=S_{p}(\ldots) /(2 S+1)$, отнюдь не может быть представлено как сум ма произведений типа

$$
<T\left(S^{i}\left(t_{1}\right) S^{k}\left(t_{2}\right)\right)>\left\langleT \left( S^{l}\left(t_{3}\right) S^{m}\left(t_{n}\right)>\ldots+\ldots\right.\right.
$$

Ввиду этого для расчета эффектов, в которых существенна некоммутативность проекции $\hat{\vec{S}}$ нужна особая техника.

Ряд вариантов такой техники для спина $S=\frac{1}{2}$ было построено несколько лет назад И.Е. Дзялошинским и автором настоящей статьи (неопубликовано). Мы здесь воспользуемся одним из этих вариантов, который нам удалось обобщить на случай произвольного спина.

Введем операторы фиктивного фермионного поля $a_{n \beta}$ и $a_{n \beta}^{+}$соответствующие оператору спина $\hat{\vec{S}}_{\mathrm{n}}$ согласно формуле

$$
a_{n \beta}^{+} \vec{S}_{\beta \beta^{\prime}} a_{n \beta^{\prime}}=\hat{\vec{S}}_{n}
$$

где $S_{\beta \beta}^{1}$ - матрицы спина. Каждый ндекс $\beta$ и $\beta^{\prime}$ пробегает $2 S+1$ значении: $-S, \ldots, S$. Операторы $\hat{S}^{1}$, введенные согласно (2), обладают правильными коммутационными соотношениями при обычных коммутационных соотношениях операторов $a_{n \beta}$

$$
\left\{\begin{array}{ll}
a_{n \beta}^{+} & a_{m \beta^{\prime}}
\end{array}=\delta_{\beta \beta^{\prime}} \delta_{m n} \quad\left\{a_{n \beta} a_{m \beta^{\prime}}\right\}=0\right.
$$


Однако они обладают одним существенным недостатком. Дело в том, что операторы $\alpha_{\beta}$ вводят фиктивные состояния. Действительно, предположим, что спин $\vec{S}$ имеет проекцию $M$ на избранную ось. Этому соответствует состояние $\Phi_{M}$, для которого $a_{M} \Phi_{M} \neq 0, a_{\beta \neq M} \Phi_{M}=0$, т.е. есть лишь одна «частица» соответствующая оператору $a_{\mathrm{M}}$ и нет «частиц», соответствующих операторам. $a_{\beta \neq M} \cdot$ Однако совершенно понятно, что введя операторы $a_{\beta}$ мы ввели в рассмотрение нефизические состояния, в которых либо вообще все числа заполнения равны нулю, либо больше одного из них равно единице. Эти состояния необходимо ИСКЛюЧИТ Б .

В случае спина $S=\frac{1}{2}$ это происходит автоматически следующим образом. Все физические величины содержат только средние от произведений операторов $\hat{S}^{1}$. Другие комбинации $a_{\beta}$ в них не входят. Но сами операторы $\hat{S}^{1}$ для $S=\frac{1}{2}$ обладают тем свойством, что дают нуль, действуя на нефизические состояния (0.0) или (1.1). Таким образом, можно. брать шпур по всем состояниям. Правда, при этом надо учесть условие нормировки. Поскольку при полном усреднении шпур делится на $4^{n}$, а при физическом только на $2^{n}$, где $n$ - число различных атомов, фигурирующих в рассматриваемом среднем, то результат надо умножить на $2^{\text {n }}$.

Эта процедура легко обобщается на случай $S>\frac{1}{2}$. Операторы (2) в этом случае дают нуль при действии на состояние $(0,0 \ldots 0)$, но отличный от нуля результат при действии на состояния, где несколько чисел заполнения равны единице. Ввиду этого мы будем считать, что на каждую «частицу» приходится энергия $\lambda \gg T$. При этом главный вклад дадут состояния, в которых $\Sigma_{\beta} N_{\beta}$ имеет минимум (т.е. 1). Эта процедура тоже требует правильной нормировки для того, чтобы среднее вида $(2 S+1)^{-1} S p\left(S^{\mathrm{i}} \hat{S}^{\mathrm{n}} \ldots\right)$ (для одного атома) имело правильное значение. Надо умножить значение, вычисленное по нашей процедуре на $\epsilon^{\lambda / T} /(2 S+1)$ и потом положить $\lambda \rightarrow \infty$. В общем случае надо умножить результат на $\left[\epsilon^{\lambda / T} /(2 S+1)\right]^{n}$, где $n$ - число участвующих атомов примеси .

Вот эта нормировка приводит фактически к существенному неудобству при пользовании изложенной техникой. Дело в том, что в противоположность обычной технике теории поля, средние от операторов, относящихся к одному атому, и от операторов для разных атомов, входят не на равных правах. Это очень существенно для задач, связанных с упорядочением спинов. Однако в рассматриваемом случае каждый атом примеси фактически рассеивает электроны независимо (это будет продемонстрировано ниже), и потому можно рассматривать лишь один атом. Тогда нормировка не вносит никаких трудностей.

Мы пользуемся температурной техникой теории пюля. «Свободные» гриновские функции для операторов $a_{\beta}$ (для одного атома) имеют в этом случае вид:

$$
\begin{aligned}
& \mathscr{G}_{\beta \beta^{\prime}}\left(\tau-\tau^{\prime}\right)=-\left\langle T\left(a_{\beta}(\tau) a_{\beta}^{+}\left(\tau^{\prime}\right)\right)\right\rangle \\
& \mathscr{G}_{\beta \beta^{\prime}}(\tau)=T \sum_{\omega} \mathscr{G} \beta \beta^{\prime(\omega)} e^{-i \omega \tau} \\
& \mathscr{G}_{\beta \beta^{\prime}}(\omega)=\delta_{\beta \beta^{\prime}} /(i \omega-\lambda), \quad \omega=(2 n+1) \pi T
\end{aligned}
$$


(среднее относится здесь ко всем состояниям). Функции $\mathscr{G}$ на диаграммах мы будем обозначать пунктирной линией.



Рис. 1

Вычислим для примера простейшие диаграммы собственной энергии электрона. Помимо иллюстрации, это вычисление позволяет предугадат наиболее важные моменты полного расчета. Рассмотрим сначала случай одного примесного атома в точке $\vec{r}$. Гамильтониан взаимодействия дается форормулой (I). Диаграмма первого неисчезающего приближения приведена на рис.1. Ей соответствует выражение

$$
\begin{aligned}
& \sum_{\alpha \alpha^{\prime}}^{(1)}\left(\vec{p}, \vec{p}^{\prime}, \omega\right)=-\frac{e^{\lambda / T}}{2 S+1}\left(\frac{J}{N}\right)^{2} S p\left(S_{i} S_{k}\right)\left(\sigma_{i} \sigma_{k}\right) \alpha \alpha^{\prime} e^{i\left(\vec{p}-\vec{p}^{\prime}\right) \vec{r}} \times \\
& \times \int d \vec{p}^{\prime \prime} /(2 \pi)^{3} T^{2} \sum_{\omega_{1} \omega_{2}} \frac{1}{i\left(\omega+\omega_{1}-\omega_{2}\right)-\xi\left(\vec{p}^{\prime \prime}\right)} \times \frac{1}{i \omega_{1}-\lambda} \times \frac{1}{i \omega_{2}-\lambda}
\end{aligned}
$$

где $\boldsymbol{\xi}$ - энергия электрона, отсчитанная от энергии Ферми.

Взяв суммы по $\omega_{1}$ и $\omega_{2}$, получаем вместо $\mathcal{T}^{2} \sum$ выражение

$$
-\frac{1}{4} \frac{1}{i \omega-\xi\left(\vec{p}^{\prime \prime}\right)}\left(\tanh \frac{\xi}{2 T}+\tanh \frac{\lambda}{2 T}\right)\left(\operatorname{coth} \frac{\lambda+\xi}{2 T}-\tanh \frac{\lambda}{2 T}\right)
$$

Полагая $\lambda \gg T$, получаем отсюда просто $-e^{-\lambda / \tau}\left[i \omega-\xi\left(\vec{p}^{\prime \prime}\right)\right]^{-1}$. Усредняя по положениям атома примеси $\vec{r}$, и просуммировав по всем атомам примеси, взяв интеграл по $\vec{p}^{\prime \prime}$ и отбрасывая действительную часть $\Sigma$, дающую лишь малую перенормировку химического потенциала, получаем:

$$
\begin{aligned}
& \sum_{\alpha \alpha^{\prime}}\left(\vec{p}, \vec{p}^{\prime}, \omega\right)=\delta_{\alpha \alpha^{\prime} \delta}\left(\vec{p}-\vec{p}^{\prime}\right)(2 \pi)^{3} \sum(\omega) \\
& \sum(\omega)=-\left(i / 2 \tau_{0}\right) \operatorname{sign} \omega \\
& \frac{1}{\tau_{0}}=\left(\frac{J}{N}\right)^{2} S(S+1) N_{i} \frac{p_{0} m}{\pi}
\end{aligned}
$$


где $N_{1}$ - число атомов примеси в единице объема.

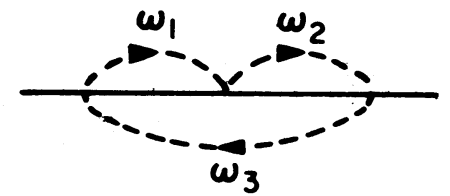

(a)

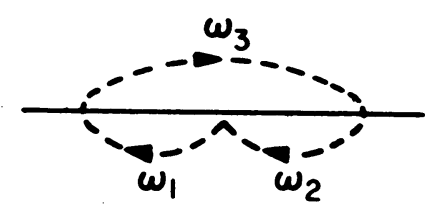

(ठ)

Рис. 2

Среди членов следующих приближений могут быть члены, зависящие только ог одного атома примеси и члены, зависящие от большого числа таких атомов. Рассмотрим прежде всего следующую поправку, зависящую от одного атома примеси. Ей соответствуют две диаграммы (рис. $2 a$ и b). Имеем для диаграммы $2 a$ (после усреднения по положениям и суммирования по всем атомам)

$$
\begin{gathered}
\sum_{\alpha \alpha^{\prime}}^{(2 a)}(\vec{p}, \omega)=-\frac{e^{\lambda / T}}{2 S+1}\left(\frac{J}{N}\right)^{3} S p\left(S_{i} S_{k} S_{l}\right)\left(\sigma_{i} \sigma_{k} \sigma_{l}\right)_{\alpha \alpha^{\prime}} \times \\
\times T^{3} \sum_{\omega_{1} \omega_{2} \omega_{3}} \int \frac{d \vec{p}^{\prime}}{(2 \pi)^{3}} \frac{d \vec{p}^{\prime \prime}}{(2 \pi)^{3}} \frac{1}{i\left(\omega+\omega_{3}-\omega_{1}\right)-\xi\left(\vec{p}^{\prime}\right)} \times \frac{1}{i\left(\omega+\omega_{3}-\omega_{2}\right)-\xi\left(\vec{p}^{\prime \prime}\right)} \times \\
\times \frac{1}{i \omega_{1}-\lambda} \times \frac{1}{i \omega_{2}-\lambda} \times \frac{1}{i \omega_{3}-\lambda}
\end{gathered}
$$

В этом выражении хорошо видны правила написания формул по диаграммам. (а) Каждой пунктирной линии сопоставляется своя частота. Электронные частоты определяются из законов сохранения. (b) Импульс электрона на каждой внутренней линии независим и по нему происходит интегрирование. (с) Берется $\alpha \alpha^{\prime}$ компонента от произведения электронных операторов спина в порядке их расположения на электронной линии. (d) Все пунктирные линии, относящиеся к одному атому, вместе составляют замкнутую петлю. Берется шпур от операторов спина примеси в порядке их расположения на петле.

Взяв суммы по $\omega_{1}, \omega_{2}$ и $\omega_{3}$ и считая $\lambda \gg T$, получаем

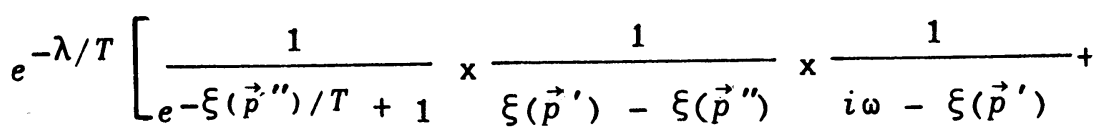

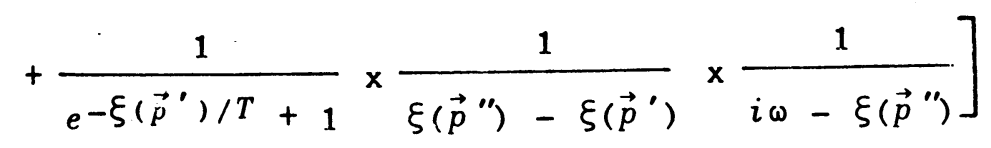


Это два совершенно симметричных выражения. Нетрудно увидеть, что первое слагаемое дает логарифмический интеграл по положительным $\xi\left(\vec{p}^{\prime \prime}\right)$ В области T, $\xi\left(\vec{p}^{\prime}\right) \ll \xi\left(\vec{p}^{\prime \prime}\right) \ll \epsilon_{\boldsymbol{F}} \cdot$ В принципе интеграл по $\vec{p}^{\prime \prime}$ распространяется на большую область. Фактически он ограничен лишь пределом, обратным радиусу взаимодействия; последний может быть гораздо меньше $1 / p_{\circ}$ для $d$ или $f$-оболочек. Однако, как будет показано в разделе 3 , эта неприятность ликвидируется перенормировко при переходе от борновской амплитуды $J$ к истинной $J_{1}$ Что касается оставшегося интеграла, то он опять берется по $\xi\left(\vec{p}^{\prime \prime}\right) \sim \omega$. Таким образом в диаграмме 2 а один из интегралов по $\xi(\vec{p})$ может быть логарифомическим, и при этом другой обязательно нелогарифмический. Поскольку логарифмическим может быть любой из интегралов, то ıозникают два одинаковых члена.

В целом получаем с логарифмической точностью

$$
\sum^{(2 a)}=\frac{i}{2 \tau_{0}} \sin \omega \frac{2 J}{N} \frac{p_{0} m}{2 \pi^{2}} \ln \frac{\varepsilon_{F}}{|\omega|}
$$

Таким же образом можно получить, что $\Sigma^{(2 \mathrm{~b})}$, соответствующая диаграмм (2b) равно $\Sigma^{(2 a)}$. В сумме полученный результат можно интерпретировать как изменение величины $1 / \tau_{0}$

$$
\frac{1}{\tau_{0}} \rightarrow \frac{1}{\tau_{0}}\left(1-\frac{4 J}{N} \frac{p_{0} m}{2 \pi^{2}} \ln \frac{\varepsilon_{F}}{\mid \omega !}\right)=\frac{1}{\tau_{0}}\left(1-\frac{3 z J}{\varepsilon_{F}} \ln \frac{\varepsilon_{F}}{|\omega|}\right)
$$

( $z$ - число электронов на 1 атом). В этом фактически и заключается результат, полученный Кондо [1] другим способом.

Анализируя проделанные выше вычисления нетрудно увидеть, что их можно описать следующим образом. В каждом из рис. 2а и 2 р мы можем провести два поперечных разреза через одну электронную и две спиновые линии. Проведем один такой разрез. В двух суммах и одном интеграле, соответствующих такому разрезу существенны значения переменных порядка $\omega$. Оставшаяся сумма и интеграл приводятся к логарифмическому интегралу, в котором существенны значения переменных в интервале $|\omega| \leqslant\left|\omega_{1}\right|,\left|\xi_{1}\right| \ll \epsilon_{\boldsymbol{F}}$. В каждой диаграмме надо проводить все возможные разрезы по трем линиям. Можно произвести анализ диаграмм следующих порядков. Если сохранять члены с максимальной степенью логарифма при данной степени $J$ (это члены $J^{\text {n+2 }} I^{\mathrm{n}} \frac{\epsilon_{F}}{\mid \omega !}$ ), то мы обязательно приходим к выводу, что это диаграммы, в которых интеграл по разрезу берется при $|\xi|,\left|\omega_{1}\right| \sim|\omega|$, a во всех остальных интегралах возникают логарифмы и существенные значения переменных $\left|\xi_{1}\right|,\left|\omega_{1}\right| \gg|\omega|$. Это положение очень напоминает рассмотрение электронного затухания в Ферми-жидкости, проведенное Г.М.Элиашбером [4], И мы воспользуемся поэтому его методом.

Однако, прежде чем это сделать, рассмотрим диаграммы следующих порядков, в которых участвует не один, а два атома примеси. Низшие диаграммы такого рода изображены на рис.за и $3 \mathrm{~b}$. Если применять усреднение по положениям примеси, то нетрудно увидеть, что вклад диаграммы $3 а$ по отношению к диаграмме 1 составляет $1 / \tau_{0} \epsilon_{\boldsymbol{F}}$, т.е. очень мал. 
Это же относится ко всем другим диаграммам с пересечением линий от разных атомов. Что же касается. диаграмм с. внутренними вставками типа 3b, то здесь можно рассуждать следующим образом. Согласно предыдущему, роль внутренней вставки (см.(5)) сводится к замене $G=(i \omega-\xi)^{-1}$ на $G^{\prime}=\left(i \omega+\frac{i}{2 \tau} \operatorname{sign} \omega-\xi\right)^{-1}$. Произведем в диаграмме $3 \mathrm{~b}$

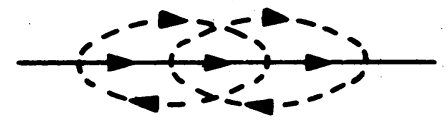

(a)



$(\delta)$

Рис. 3

замену $G^{\prime}=G+\left(G^{\prime}-G\right)$ и в члене $c G^{\prime}-G$ будем интегрировать сначала по $\xi$. При этом получается нуль. Значит остается только интеграл $c G$. Это относится ко всем диаграммам с внутренними вставками. Итак, достаточно рассмотреть лишь диаграммы для одного атома примеси .

\section{2. Собственно-энергетическая часть}

Как уже сказано, мы применим метод работы [4]. В любой из интересующих нас диаграмм мы можем провести разрез по трем линиям. Суммы и интегралы по этим переменным надо вычислять точно, так как

здесь существенны малые значения аргументов. Что касаетя остальных частей диаграммы, то в них существенны большие значения переменных, и, с логарифомической точностью, мы можем заменить все суммы по частотам интегралами. Таким образом, нам предстоит вычислить полную

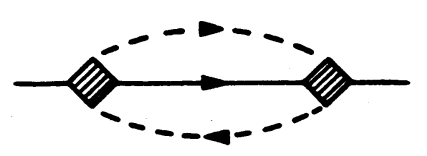

Рис. 4

сумму диаграмм, имеющую вид изображенный на рис.4, где заштрихованные квадраты обозначают сумму всех «четыреххвосток», вычисленных с логарифмической точностью. Мы ее обозначим

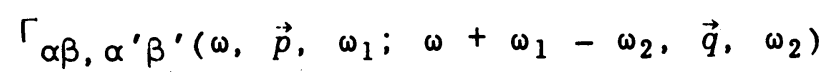




$$
-\frac{e^{\lambda / T}}{2 S+1} N_{i} T^{2} \sum_{\omega_{1} \omega_{2}} \int \frac{d \vec{q}}{(2 \pi)^{3}} \Gamma_{\alpha \beta, \alpha^{\prime \prime} \beta} \cdot\left(\omega, \vec{p}, \omega_{1} ;-\omega+\omega_{1}-\omega_{2}, \vec{q}, \omega_{2}\right) \times
$$

$$
\times \Gamma_{\alpha^{\prime \prime} \beta^{\prime}, \alpha^{\prime} \beta}\left(\omega+\omega_{1}-\omega_{2}, \vec{q}, \omega_{2} ; \omega, \vec{p}, \omega_{1}\right) G\left(\omega+\omega_{1}-\omega_{2}, \vec{q}\right) \mathscr{G}\left(\omega_{1}\right) \mathscr{G}\left(\omega_{2}\right)
$$

где $\quad G(\omega, \vec{p})=(i \omega-\xi(\vec{p}))^{-1}, \mathscr{G}(\omega)=(i \omega-\lambda)^{-1}$. Мы увидим в дальнейшем что $\Gamma$ будет зависеть только от $\ln \frac{\epsilon_{\boldsymbol{F}}}{|\omega|}$. Ввиду этого мы можем произвести переход от суммирования по мнимым частотам к интегрированию по действительным и перевести $\omega$ на действительную ось. Из соображений симметрии следует, что спиновая зависимость Г имеет Вид

$$
\Gamma=\Gamma^{(0)}+\Gamma^{(\vec{\sigma})}(\vec{\sigma} \vec{S})
$$

Аналогично [4] имеем

$$
\begin{gathered}
\frac{1}{2 S+1} \Gamma_{\alpha \beta, \alpha^{\prime \prime} \beta^{\prime}}(1,2 ; 3,4) \Gamma_{\alpha^{\prime \prime} \beta^{\prime}, \alpha^{\prime} \beta}(3,4 ; 1,2)= \\
\quad=\left[\left|\Gamma^{(0)}\right|^{2}+S(S+1)\left|\Gamma^{(0)}\right|^{2}\right] \delta_{\alpha \alpha^{\prime}}
\end{gathered}
$$

$[4] \begin{array}{r}\text { Производя } \\ \text { получаем }\end{array}$

$$
\begin{gathered}
T^{2} \sum_{\omega_{1} \omega_{2}} G\left(\omega+\omega_{1}-\omega_{2}, \dot{\vec{q}}\right) \mathscr{G}\left(\omega_{1}\right) \mathscr{G}\left(\omega_{2}\right)= \\
=\frac{i}{(2 \pi)^{2}} \int \operatorname{Im} \mathscr{G}_{R}\left(\omega_{1}\right) \operatorname{Im} \mathscr{G}_{R}\left(\omega_{2}\right) \operatorname{Im} G_{R}\left(\omega+\omega_{1}-\omega_{2}\right) \times \\
\times\left(\tanh \frac{\omega_{2}}{2 T}-\operatorname{coth} \frac{\omega_{2}-\omega}{2 T}\right)\left(\tanh \frac{\omega_{1}}{2 T}-\tanh \frac{\omega+\omega_{1}-\omega_{2}}{2 T}\right) d \omega_{1} d \omega_{2}
\end{gathered}
$$

Здесь учтено, что интегралы должны браться при малых значениях $\omega_{1}, \omega_{2}\left(\right.$ ччитаем $\left.T \ll \lambda \ll \epsilon_{F}\right)$. ПодставляЯ $\operatorname{Im}_{R}(\omega)=-\pi \delta(\omega-\xi)$,

$$
\sum=-i \operatorname{sign} \omega N_{i} \frac{p_{0} m}{2 \pi}\left[\left.\Gamma^{(0)}(\omega, \lambda ; \omega, \lambda)\right|^{2}+S(S+1)\left|\Gamma^{(\sigma)}(\omega, \lambda ; \omega, \lambda)\right|^{2}\right]
$$

отличающееся от первого приближения (см. (5)) заменой $(J / N)^{2} S(S+1)$ на квадратную скобку.

Теперь будем рассматривать Г. Как уже отмечено, Г можно вычис- 
лять так же, как при $T=0$, заменив все суммы по частотам интегралами. Но это еще интегралы по мнимой оси. Автором настоящей статьи было доказано (неопубликовано), что все эти интегралы можно перевести на действительную ось, вместе с внешними аргументами. При этом все температурные Гриновские функции надо заменить на временные при $T=0$, путем перехода $i \omega \rightarrow \omega+i \delta \operatorname{sign} \omega$. После этого сделаем следующее. Интегралы по $\omega_{1}$ идут только по частотам спиновых линий. Ввиду того, что все эти интегралы берутся от $-\infty$ до $\infty$, мы можем сделать замену $\omega-\lambda \rightarrow \omega$. Поскольку в каждой элементарной вершине есть одна входящая и одна выходящая спиновая линия, то законы сохранения при этом не нарушатся. Надо только сделать замену и внешних частот $\omega-\lambda$ на $\omega$.

Поскольку нам надо найти $\Gamma(\omega, \lambda ; \omega, \lambda)$ то, после всех замен дело сводится к вычислению $\Gamma(\omega, 0 ; \omega, 0)$ в обычной временной диаграммной технике при $T=0$, причем в качестве $G$ - функций надо брать $(\omega-\xi+i \delta \operatorname{sign} \xi)^{-1}$, а в качестве $\mathscr{G}$ функций $(\omega+i \delta)^{-1}($ т.к. $\lambda>0)$.

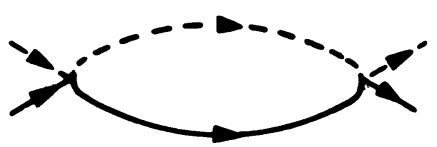

(a)

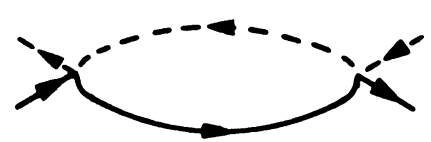

$(\delta)$

Рис. 5

Первые диаграммы для вершинной части имееют вид, изображенный на рис. 5. Получаем для них следующие выражения (поправки $\mathrm{\kappa} \mathrm{J} / \mathrm{N}$ ):

$$
\begin{gathered}
-i(J / N)^{2}(\vec{\sigma} \vec{S})^{2} \frac{p_{0} m}{2 \pi^{2}} \int \frac{d \omega_{1}}{2 \pi} \int d \xi_{1} \frac{1}{\omega_{1}+i \delta} \times \frac{1}{\omega-\omega_{1}-\xi_{1}+i \delta \operatorname{sign} \xi_{1}}= \\
\quad=(J / N)^{2} \frac{p_{0} m}{2 \pi^{2}}[S(S+1)-\vec{\sigma} \vec{S}]\left[\ln \frac{\varepsilon_{F}}{|\omega|}+i \pi \theta(\omega)\right] \\
-i(J / N)^{2} \sigma^{i} \sigma^{k} S^{k} S^{i} \frac{p_{0} m}{2 \pi^{2}} \int \frac{d \omega_{1}}{2 \pi} \int d \xi_{1} \frac{1}{\omega_{1}+i \delta} \times \frac{1}{\omega+\omega_{1}-\xi_{1}+i \delta \operatorname{sign} \xi_{1}}= \\
=-(J / N)^{2} \frac{p_{0} m}{2 \pi^{2}}[S(S+1)+\vec{\sigma} \vec{S}]\left[\ln \frac{\varepsilon_{F}}{|\omega|}+i \pi \theta(-\omega)\right]
\end{gathered}
$$

где $\theta(x)=\left\{\begin{array}{l}1, x>0 \\ 0, x<0\end{array}\right\}$. Мы оставили здесь и нелогарифмический мнимый член, поскольку он понадобится для дальнейших рассуждений. 
Рассматривая диаграммы высших порядков (рис. 6) нетрудно выясниты что максимальной степенью логарифма в каждом данном порядке обладаюл только диаграммы, поддающиеся последовательному упрощению двум линиям, одной электронной и одной спиновой, такому, что в результате получается элементарная вершина. Например, упрощая рис бс можно получить сначала диаграмму типа $6 a$, затем 5b и затем, простую вершину. Этим свойством обладают так называемые «паркетные» диаграммы $5 a, b ; 6 a, b, c$, и не обладает, например $6 \mathrm{~d}$.

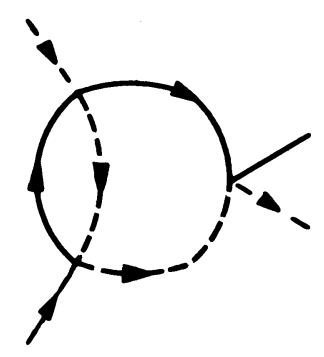

(a)

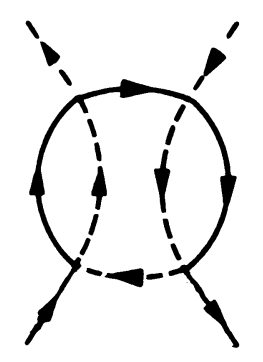

(B)

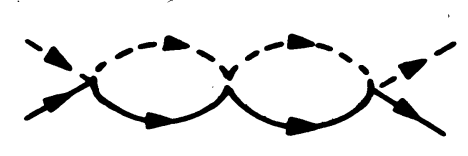

$(\delta)$

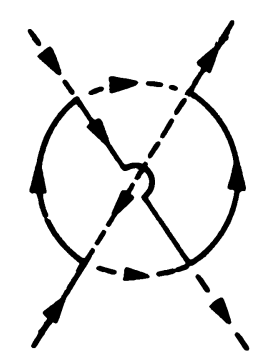

(2)

Рис. 6
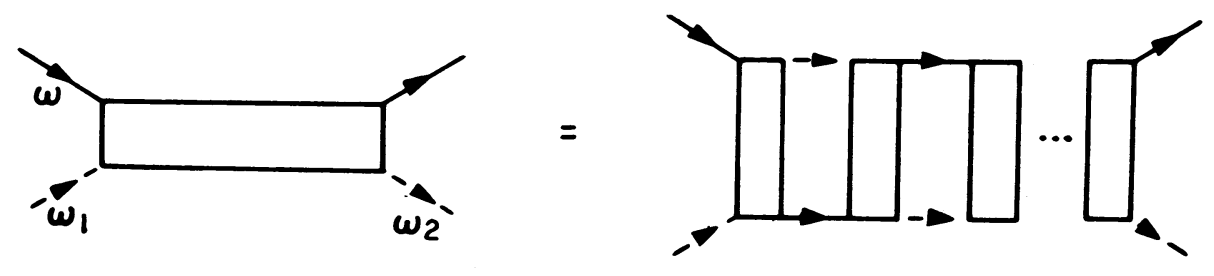

(a)
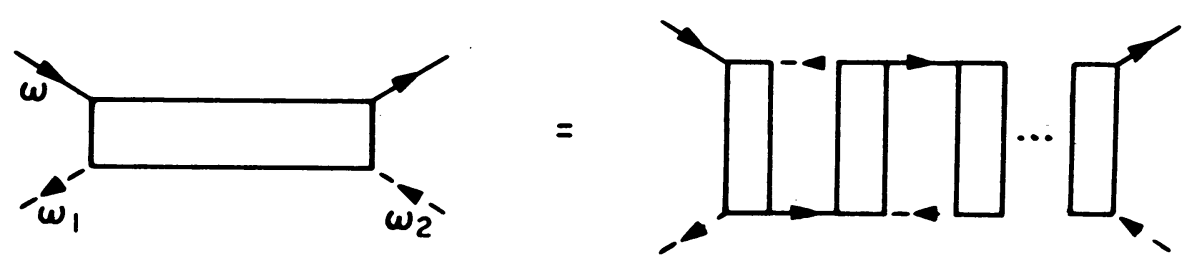

(ठ)

Рис. 7 
Мы просуммируем «паркет» по методу В.В. Судакова [5]. Отметим, что имеются два различных элемента «паркета», изображенные на рис.5а и 5b. Мы можем ввести в рассмотрение две разных суммы диаграмм, изображенные на рис. $7 \mathrm{a}$ и $\mathrm{b}$.

обозначим диаграмму, разрезаемую по двум параллельным линиям (рис. 7a), через $\Lambda_{1}$ и диаграмму, разрезаемую по двум антипараллельным линиям, через $\Lambda_{2}$ (рис.7b). Очевидно, что $\Gamma=\Gamma_{0}+\Lambda_{1}+\Lambda_{2}$, Где $\Gamma_{0}$ - «затравочное» взаимодействие $J / N(\vec{\sigma} \vec{S})$. Заметим, что в логарифмическом интегрировании во внутренних $\Lambda_{1}, \Lambda_{2}$ на рис. $7 а$ и b участвуют значения аргументов, большие, чем их внешние концы, т.е. чем соответствуюие пары из двух параллельных или двух антипараллельных линий .

Рассмотрим теперь для определенности диаграмму 7 а для $\Lambda_{1}$. Очевидно, что самым нижним пределом лргарифмических интегралов в этой диаграмме является $\omega$ (если внешние $\left.\omega_{1}=\omega_{2}=0\right)$. Выделим ту из внутренних пунктирных линий, у которой $\omega_{1}$ минимально. Слева и справа от этого элемента могут стоять любые элементы $\Gamma$, т.е. иначе говоря, в сумме и слева, и справа стоит полная $\Gamma$. Роль $\omega$ для этих $\Gamma$ играет величина $\omega_{1}$. Таким образом, можно написать уравнение (с логарифммической точностью):

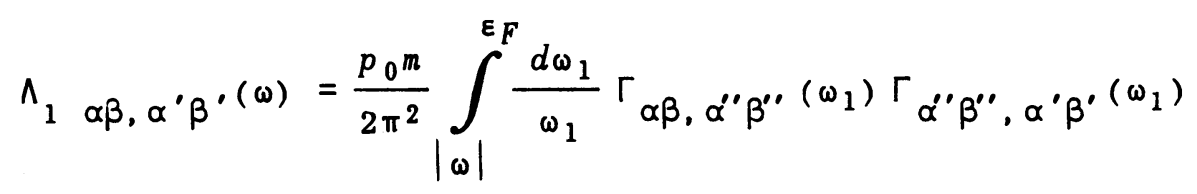

Аналогичным образом

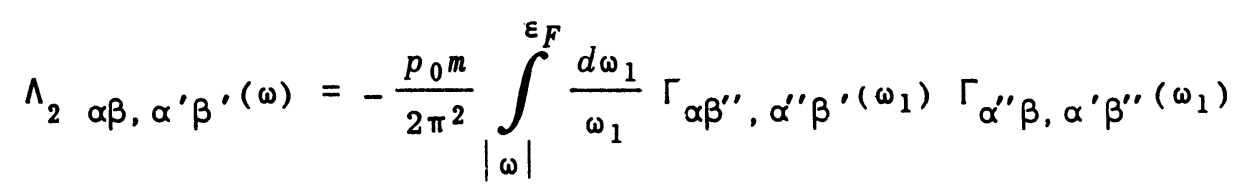

Складывая выражения, получаем

$$
\begin{aligned}
& \Gamma_{\alpha \beta, \alpha^{\prime} \beta^{\prime}(\omega)}=\frac{J}{N}(\vec{\sigma} \vec{S})+\frac{p_{0} m}{2 \pi^{2}} \int_{|\omega|}^{\varepsilon_{F}} \frac{d \omega_{1}}{\omega_{1}}\left[\Gamma_{\alpha \beta, \alpha^{\prime \prime} \beta^{\prime \prime}\left(\omega_{1}\right)} \Gamma_{\alpha^{\prime \prime} \beta^{\prime \prime}, \alpha^{\prime} \beta}\left(\omega_{1}\right)-\right. \\
& \left.\Gamma_{\alpha \beta^{\prime \prime}, \alpha^{\prime \prime} \beta^{\prime}\left(\omega_{1}\right)} \Gamma_{\alpha^{\prime \prime} \beta, \alpha^{\prime} \beta^{\prime \prime}}\left(\omega_{1}\right)\right]
\end{aligned}
$$

Введем переменную $\ln \frac{\epsilon_{\boldsymbol{F}}}{|\omega|}=x$ и будем искать решение в виде $\Gamma=\Gamma^{(0)}+(\overrightarrow{\sigma \vec{S}}) \Gamma^{(\sigma)}$. При этом получаем:

$$
\begin{aligned}
\Gamma^{(\sigma)}(x) & =\frac{J}{N}-\frac{p_{0} m}{\pi^{2}} \int_{0}^{x} d y\left[\Gamma^{(\sigma)}(y)\right]^{2} \\
\Gamma^{(0)} & =0
\end{aligned}
$$


Решая интегральное уравнение, получаем:

$$
\Gamma=\frac{(J / N) \vec{\sigma} \vec{S}}{1+\frac{J}{N} \frac{p_{0} m}{\pi^{2}} \ln \frac{\varepsilon_{F}}{|\omega|}}
$$

Подставляя в $(9)$, получаем

$$
\sum=-\frac{i}{2 \tau_{0}} \sin \omega\left[1+\frac{3 J z}{2 \varepsilon_{F}} \ln \frac{\varepsilon_{F}}{|\omega|}\right]^{-2}
$$

В выражениях (14) и (15) имеется одна особенность. Если $J<0$, то при таком значении $|\omega|$ эти выражения имеют полюс. Наличие такого полюса свидетельствует о том, что в рассеянии имеется резонанс Учет конечной ширины резонанса должен привести к тому, что в знаменателе формулы (14) и к логарифмическому члену прибавится мнимое слагаемое пропорцональное $\frac{i J}{N} \frac{p_{0} m}{\pi^{2}}$. Это видно уже из формулы (10). Для нахождения таких членов наше приближение неприменимо. Поэтому мы будем пользоваться выражениями (14), (15) вдали от полюса, a в окрестности полюса оценивать лишь порядки величины, основываясь на идее, что $к$ ln $\frac{\epsilon_{\boldsymbol{F}}}{|\omega|}$ в знаменателе прибавляется слагаемое, пропор-
циональное $i \pi$.

3. Обычное взаимодействие. Переход к амплитуде рассеяния

До сих пор мы ограничивались рассмотрением обменного взаимодеиствия. В то же время во взаимодействии электрона с атомом примеси обязательно присутствует обычный необменный член, значительно бо́льшей величины. Естественно, возникает вопрос о возможности интерференционных эффектов .

Обычное взаимодействие, отличное от обменного, может рассматриваться, как внешнее поле и обозначается крестом на электронной линии •

Прежде всего отметим, что по тем же причинам, что и раньше, доста точно рассматривать взаимодействие только с одним атомом. Теперь предположим, что в диаграмме $5 \mathbf{a}$ у нас на электронной линии вставлено некоторое количество крестов (рис. 8). Считая для простоты взаимодействие изотропным, получаем

$$
-\frac{i}{2 \pi}\left(\frac{J}{N}\right)^{2}[S(S+1)-\vec{\sigma} \vec{S}]\left(\frac{p_{0} m}{2 \pi^{2}}\right)^{2} \Phi \int d \omega_{1} d \xi_{1} d \xi_{2} \frac{1}{\omega_{1}+i \delta} \frac{1}{\omega-\omega_{1}-\xi_{1}+i \delta \sin \xi_{1}} \times
$$

$$
x \frac{1}{\omega-\omega_{1}-\xi_{2}+i \delta \sin \xi_{2}}
$$


где через $\varphi$ обозначена вся вставка от первого креста до последнего. Интегрируя, можно убедиться, что логарифмическое выражение в этом случае не получается. Учитывая, что сумма всех возможных $\varphi$ имеет порядок $\frac{\epsilon_{F}}{N}$, находим, что диаграмма со вставкой в $1 / \ln \frac{\epsilon_{F}}{|\omega|}$ раз меньше диаграммы без вставки .

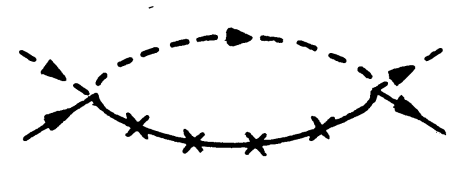

Рис. 8

Однако крест можно вставить и в ту электронную линию, которая не участвует в логарифмических интегралах. Проще всего вставить кресты в самую первую диаграмму la При этом можно убедиться, что диаграмма со вставкой имеет порядок $\frac{\omega}{\epsilon_{F}} \cdot$ Отсюда следует, что обычное взаимодействие дает отдельное слагаемое в $\Sigma$ и никак не интерферирует с обменной частью. Обычная часть была рассмотрена в целом ряде работ (см. например [3], \$39.2). Она имеет вид, где

$$
-\frac{i}{2 \tau_{\text {ora }}} \operatorname{sign} \omega, \text { где } \frac{1}{\tau_{\text {ord }}}=N_{1} \frac{m p_{0}}{\pi}|a|^{2}
$$

( $a$ - амплитуда рассеяния, соответствующая необменному взаимодействИю) .

Теперь рассмотрим второй существенный вопрос. Во всем предыдущем расчете интегралы по $\xi$ брались в пределах $-\epsilon_{\boldsymbol{F}}<\xi<\epsilon_{\boldsymbol{F}}$. В действительности эти интегралы со стороны положительных $\xi$ ограничены только условием $|\vec{p}|<\frac{1}{r_{\text {Int }}}$, где $r_{\text {int }}$ - радиус взаимодействия, который может быть гораздо менььше $1 / p_{0}{ }^{1 n t} \cdot$ Ввиду этого, надо произвести перенормировку взаимодействия.

Вместо борновской амплитуды рассеяния мы введем истинную обменную амплитуду рассеяния әлектрона на атоме. Если $\hat{f}$ есть борновская амплитуду рассеяяния әлектрона на атоме. Если $\hat{f}_{1}$ - истинная, то аналогично $3 \$ 25.4$ имеем:

$$
\hat{f}=\hat{f}_{1}-\hat{f} \hat{f}_{1} \frac{p_{0} m}{2 \pi^{2}} \int \frac{d \xi_{1}}{\xi-\xi_{1}+i \delta}
$$

Роль $\hat{f}$ до сих пор играла у нас величина $-\frac{\mathcal{J}}{N} \vec{\sigma} \vec{S}$ Но мы теперь будем считать $\hat{f}=-\frac{J}{N} \vec{\sigma} \vec{S}+f_{0}$ где $f_{0}$ не зависит от спинов, и подберем $f_{0}$ так, чтобы уравнение (16) удовлетворялось при $\hat{f}_{1}=-\frac{J_{1}}{N} \vec{\sigma}_{S}$. 
Это означает, что в «затравочное» взаимодействие мы включили часть обычного взаимодействия. Диаграммная техника от этого не страдает, так как оператор $f_{0} a_{\beta}^{+} a_{\beta}$. тоже дает нуль, действуя на состояние с нулевыми числами заполнения, а другие неправильные состояния исключаются примененной нами процедурой. Это обстоятельство, вообще говоря, дает возможность рассматривать обычное взаимодействие вместе с обменным. - Однако, поскольку обычная часть, кроме перенормировки, не дает никакого вклада в логарифмические интегралы (вклад обычного взаимодействия в интегралы (10) (a) и (b) взаимно сокращается), то мы рассмотели его отдельно. Это имеет и то-преимущество, что обычное взаимодействие $U$, как правило сильнее обменного и поэтому совместное рассмотрение привело бы к совершенно ненужному ограничению $(J / N) \ln \frac{\epsilon_{\boldsymbol{F}}}{T} \gg U$. Включаемая часть. $f_{0}$, как мы сейчас увидим, имеет порядок $\frac{J}{N} \frac{J}{\epsilon_{F}}$ и не приводит к новым ограничениям.

Решая уравнение (16) с точностью до второго порядка, имеем

$$
\hat{f}=\frac{J_{1}}{N} \vec{\sigma} \vec{S}-\left(\frac{J_{1}}{N}\right)^{2} \frac{p_{0} m}{2 \pi^{2}}[S(S+1)-\vec{\sigma} \vec{S}] \int \frac{d \xi_{1}}{\xi-\xi_{1}+i \delta}
$$

При нахождении членов второго порядка (формула (10)) мы производим перенормировку следующим образом. Заменяем в (10) $J$ на $J_{1} \cdot$

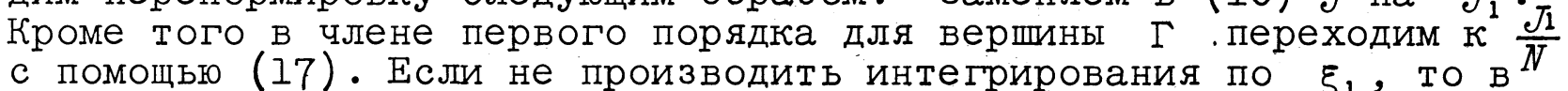
сумме получаем:

$$
\left(\frac{J_{1}}{N}\right)^{2}[S(S+1)-\vec{\sigma} \vec{S}] \frac{p_{0} m}{2 \pi^{2}} \int d \xi_{1}\left[\frac{\theta\left(\dot{\xi}_{1}\right)}{\xi_{1}-\omega-i \delta}-\frac{1}{\xi_{1}-\xi+i \delta}\right]
$$

В дальнейшем мы убедимся, что для нахождения проводимости будет нужен случай $\xi<\epsilon_{\boldsymbol{F}}$. При этом получаем с логариф̆мической точностью

$$
-\left(\frac{J_{1}}{N}\right)^{2}[S(S+1)-\vec{\sigma} \vec{S}] \frac{p_{0} m}{2 \pi^{2}} \int d \xi_{1} \frac{\theta\left(-\xi_{1}\right)}{\xi_{1}-\omega}=\left(\frac{J_{1}}{N}\right)^{2} \frac{p_{0} m}{2 \pi^{2}}[S(S+1)-\vec{\sigma} \vec{S}] \ln \frac{\varepsilon_{F}}{|\omega|}
$$

Поскольку теперь интегрирование идет по отрицательным $\xi_{1}\left(|\vec{p}|<p_{0}\right)$, то вопрос о пределе интеграла не возникает.

Так обстоит дело в низшем приближении. Покажем, что такая же перенормировка может быть произведена и в высших приближениях. Легче всего это сделать переходя от $n$-го порядка $\kappa n+1$-му. Этот переход может быть осуществлен путем поочередной замены каждой простой вершины на сумму двух вершинных частей, изображенных на рис.5а и 5b.

Если в $n$-ом порядке во всех вершинах стояли $J_{1} / N$, то в новых диаграммах $n+1$-го порядка при этой замене тоже везде станут $J_{1} / N$. Но кроме этого надо усовершенствовать саму диаграмму $n$-го порядка, подставляя поочередно, вместо всех $J_{1} / N$ выражение (17). Иными словами при таком переходе от $n-г о \kappa n+1$-му приближению интегралы типа (IOa) заменяются на $\left(10 a^{I}\right)$. Если к тому же учесть, что во всех логарифмических интегралах $\omega \sim \xi$, то практически дело сводится к тому, что во всех вершинах становятся $J_{1} / N$ и все интегралы 
берутся по области $\xi<0$, т.е. можно брать $\epsilon_{\boldsymbol{F}}$ в качестве предела интегралов по $\xi$.

\section{4. Сопротивление}

Как известно (см. например [3], \$ 39.3) связь между током и векторным потенциалюм, зависящим от частоты, при отсутствии пространственной дисперсии имеет следующий вид

$$
j_{i}(\omega)=Q_{i k}(\omega) A_{k}(\omega)
$$

причем для случая изотропного рассеяния $Q(\omega)$ представляет собой аналитическое продолжение на действительную ось $\omega$ выражения:

$$
\frac{N e^{2}}{m c} \delta_{i k}+\frac{2 e^{2}}{(2 \pi)^{3} m^{2} c} T \sum_{\omega^{\prime}} \int d \vec{p} p_{i} p_{k} G\left(\vec{p}, \omega^{\prime}\right) G\left(\vec{p}, \omega^{\prime}-\omega\right)
$$

где $G$-температурные гриновские функции. Перейдем от суммирования по $\omega^{\prime}$ к интегрированию по действительной частоте (см. [3], § 21.2), после чего (считая в (19) $w>0$ ) аналитически продолжим это выражение на действительные ш. Получаем при этом:

$$
\begin{gathered}
\frac{N e^{2}}{m c} \delta_{i k}+\frac{e^{2}}{\pi(2 \pi)^{3} m^{2} c} \int_{-\infty}^{\infty} d \omega^{\prime} \int d \vec{p} p_{i} p_{k} \operatorname{Im} G_{R}\left(\vec{p}, \omega^{\prime}\right) \times \\
\times\left[G_{A}\left(\vec{p}, \omega^{\prime}-\omega\right)+G_{R}\left(\vec{p}, \omega^{\prime}+\omega\right)\right] \tanh \frac{\omega^{\prime}}{2 T}
\end{gathered}
$$

Ввиду того, что фуннции $G$ не зависят от направления $\vec{p}$ и интегралы по $\vec{p}$ берутся в окрестности Ферми-поверхности, можно сделать замену

$$
\int \frac{d \vec{p}}{(2 \pi)^{3}} p_{i} p_{k}-\frac{1}{3} \delta_{i k} \frac{p_{0}^{3} m}{2 \pi^{2}} \int d \xi
$$

Интегрирование должно осуществляться сначала по $\omega$, а потом по $\xi$ • Однако мы, как всегда (см. [3]), применим следующий прием. Прибавим и вычтем из подынтегрального выражения, соответствующее ему «свободное» выражение, т.е. без взаимодействия с примесями. Тогда в интеграле со «свободным» выражением мы будем интегрировать сначала по частоте, а в сходящемся члене с разностью будем интегрировать сна-

чала по $\xi$ в

$$
\left(\omega-\xi+\frac{i}{2 \tau(\omega)}\right)^{-1}
$$




$$
\frac{1}{T(\omega)}=\frac{1}{T_{0 r d}}+\frac{1}{T_{\text {ex }}(\omega)}
$$

причем $\tau_{\text {ord }}$ необменное время столкновений, a $1 / T_{\text {ex }}(\omega)$ соответствует выражению (15). После всех операций, полагая $ш \rightarrow 0$, получаем

$$
\vec{j}=\frac{i \sigma \omega}{c} \vec{A}
$$

где

$$
\sigma=\frac{N e^{2}}{m} \int_{0}^{\infty} \frac{d \omega T(\omega)}{2 T \cosh { }_{2 T}^{2 \omega}}
$$

С логариф̆мической точностью получаем: для сопротивления

$$
\rho=\rho_{0 r d}+\rho_{e x}
$$

где

$$
\rho_{e x}=\rho_{0, e x}\left[1+\frac{3 J_{1} z}{2 \varepsilon_{F}} \ln \frac{\varepsilon_{F}}{T}\right]^{-2}
$$

где

$$
P_{0, e x}=\frac{3 \pi m J_{1}^{2} S(S+1) \mathrm{C}}{2 N \varepsilon_{F} e^{2 \hbar}}
$$

(c - атомная концентрация)

Если $J_{1}>0$, то $\rho$ уменьшается с понижением температуры и при $T \rightarrow 0$ обращается в нуль. В случае $J_{1}<0$, выражение (23) обращается в бесконечность при

$$
T_{\text {max }}=q \varepsilon_{F} \exp \left(-\frac{2 \varepsilon_{F}}{3\left|J_{1}\right| z}\right)
$$

$(q \sim 1)$. Согласно рассмотрению $\$ 2$ в действительности в окрестности этой точки сопротивление имеет максимум. В максимуме

$$
\rho_{\text {ex }} \sim \rho_{0, e x}\left(\varepsilon_{F} / J_{1}\right)^{2}
$$

Согласно (24) это выражение порядка обычного остаточного сопротивления, вызываемого теми же примесями. Ширина максимума по температуре $\Delta T \sim T_{\circ} \cdot$ При дальнейшем уменьшении температуры $\rho_{\text {ex }} \rightarrow 0$.

Мы не разбирали вопроса о влиянии ферромагнитного упорядочения. Однако, по-видимому, оно ликвидирует рассмотренный эффект. Посколь ку температура ферромагнитного перехода $T_{c} \sim c J_{1}^{2} / \epsilon_{F}(c м \cdot[2])$, где c - атомная концентрация примесей, то максимум в-принципе может быт получен при любом сочетании металлов с $J_{1}<0$ и при досаточно малой 
концентрации примесей.

В дальнейшей работе будет рассмотрено влияние примесного ферромагнетизма и внешнего магнитного поля.

Выражаю благодарность И.Е,Дзялошинскому за многочисленные полезные обсуждения.

\section{JИTEPATУPA}

1. J. Kondo, Prog. Theor. Phys., 32, 37 (1964).

2. А.А. Абрикосов, Л.П. Горьков, ЖЭТФ, 43, 2230 (1962).

3. A.A. Abrikosov, L.P. Gorkov, I.E. Dzyaloshinski, "Quantum Field Theory in Statistical Physics", Prentice Hall, 1963.

4. Г.М. Элиашберг, ЖЭтФ, 42, 1658 (1962).

5. В.В. Судаков, ДАН, 111, 338 (1956). 